\title{
Differences between predictions of how a reflection behaves based on the behaviour of an object, and how an object behaves based on the behaviour of its reflection
}

\author{
Ivana Bianchi ${ }^{\mathrm{a}, *}$, Marco Bertamini ${ }^{\mathrm{b}}$, Ugo Savardi ${ }^{\mathrm{c}}$ \\ a Department of Humanities (Section Philosophy and Human Sciences), University of Macerata, via Garibaldi, 20, 62100 Macerata, Italy

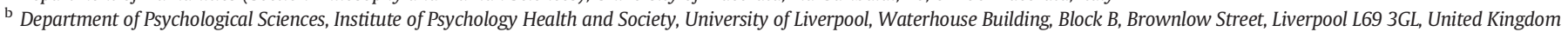 \\ c Department of Philosophy, Education and Psychology, University of Verona, Lungadige Porta Vittoria 17, 37129 Verona, Italy
}

\section{A R T I C L E I N F O}

\section{Article history:}

Received 13 June 2015

Received in revised form 17 August 2015

Accepted 20 August 2015

Available online $\mathrm{xxxx}$

\section{Keywords:}

Mirrors

Reflection

Naïve optics

Viewpoint

Identity

Opposition

\begin{abstract}
A B S T R A C T
We studied adults' understanding of the relationship between objects and their reflections. Two studies investigated whether adults performed in a similar way when asked to predict the movement of a reflection in a flat mirror based on the movement of the corresponding object or, vice versa, predict the movement of the material object based on the movement of its reflection. We used simple movements in the experiments: movements in a straight line at various angles with respect to the mirror. Despite the simplicity of the task, some of the participants made incorrect predictions in a percentage of cases ranging from $0 \%$ to $54 \%$, depending on the angle. Asymmetries between the two directions of prediction emerged, in particular in terms of types of error. Results confirmed a cognitive difference between deriving the reflected (virtual) world from the "real" (material) world and vice versa. In particular the expectation that something will be opposite in a mirror is more salient when people imagine how a reflection will be with respect to the material world rather than when they imagine how the material world will be with respect to a reflection.
\end{abstract}

(c) 2015 Elsevier B.V. All rights reserved.

\section{Introduction}

“...there's the room you can see through the glass - that's just the same as our drawing room, only the things go the other way."

[Lewis Carroll (Through the Looking Glass and What Alice Found There, 1871, p. 8)]

Mirror reflections are common phenomena in modern environments and the fascinating history of mirrors has been the subject of a number of popular books (Melchior-Bonnet, 2001; Pendergrast, 2003). We interact with mirrors on a daily basis, for instance when driving, in a gym or a dance class, or in the morning before leaving home when we glance in the mirror to see if we look alright. Interacting with mirrors requires some understanding of the correspondence between what we see in a mirror and the object that exists outside the mirror, but this understanding may be implicit or explicit. Some studies have documented the reasoning that people go through regarding the correspondence between material objects and reflections (see next section), but an issue that has not been systematically investigated is whether this correspondence is symmetrical or not: is the answer the

\footnotetext{
* Corresponding author.

E-mail addresses: ivana.bianchi@unimc.it (I. Bianchi), m.bertamini@liverpool.ac.uk (M. Bertamini), ugo.savardi@univr.it (U. Savardi).
}

same when people are asked to predict one feature of a material object based on its reflection or, vice versa, predict one feature of a reflection based on its material counterpart? From an optical and geometrical point of view the transformation is symmetrical and the question might sound silly. But studies on naïve physics (e.g. McCloskey, 1983a, 1983b; McCloskey, Washburn, \& Felch, 1983; McCloskey, Caramazza, \& Green, 1980) and naïve optics (e.g. Croucher, Bertamini, \& Hecht, 2002; Lawson \& Bertamini, 2006; Bianchi \& Savardi, 2012) have clearly demonstrated that people do not necessarily reason in terms of the physical or optical laws which they were exposed to at school (and which in many cases they only recall as explicit knowledge) when they make predictions about the trajectory or speed of moving objects or about how reflections in mirrors behave. They base their prediction on what they imagine and imagining slightly different scenarios alters their prediction. A paper which epitomizes this is Yates et al. (1988) which shows how people radically change their prediction concerning the trajectory of a moving object in situations when small changes to the imagined scenario are suggested even if the scenarios are all subject to the same physical laws. Therefore, any differences discovered between predictions about movement in a reflection based on the imagined movement of the corresponding material object and, conversely, predictions about the movement of a material object based on its reflection would help us to understand how people think of mirrors from a cognitive point of view rather than in merely optical-geometrical terms. In the present paper we describe two experiments involving 
predictions of simple movements and analyse the results. In the first experiment we used a paper and pencil task, and in the second a real-life setting.

\subsection{Predicting the correspondence between an object and its reflection}

Memory for layouts is generally very good in humans (Simons, 1996). The layout of a scene is matched by the layout of a reflected scene. Various studies have directly or indirectly addressed the issue of how people understand this relationship, but they have never taken into consideration whether the perception of this relationship is identical in both directions, i.e. from a reflection to the material world or vice versa.

\subsubsection{Correspondence of size}

People are accurate when they make judgments about the size of a material object starting from the corresponding reflection (Bianchi, Savardi, \& Bertamini, 2008; Higashiyama, 2004; Higashiyama, Shimono, \& Zaitsu, 2005; Jones \& Bertamini, 2007). The situation is very different when judging the size of an image on the surface of a mirror: predictions of the size of the reflection of one's own head, of another person's head, or the size of a simple figure are, for example, biased (Bertamini \& Parks, 2005; Lawson \& Bertamini, 2006; Lawson, Bertamini, \& Liu, 2007). However, in this study we will not consider the information on the mirror surface and focus instead on the link between material and virtual objects.

\subsubsection{Correspondence of location}

Another aspect concerns the correspondence between the position occupied in space by an object or body and that of its reflection. It has been shown that people are reasonably accurate when they judge the orthogonal distance of an object from a flat mirror surface basing their assessment on its reflection (Higashiyama \& Shimono, 2004). However, when lateral positions are involved they are less accurate. For example, $20 \%-40 \%$ of adults expect a person entering a room and moving parallel to a mirror surface to see their reflection appear at the far edge rather than the near edge of the mirror (Bertamini, Spooner, \& Hecht, 2003). Similar errors are found with movements at various angles of incidence with respect to the mirror (Savardi, Bianchi, \& Bertamini, 2010). Moreover, many adults expect to see what seems to be a slight expansion of the space directly in front of the mirror in a reflection, independent of the observer's viewpoint (Bertamini, Lawson, Jones, \& Winters, 2010; Bianchi \& Savardi, 2012; see also Croucher et al., 2002). This also holds for depicted scenes (Bertamini, Latto, \& Spooner, 2003).

\subsubsection{Correspondence of orientation}

For decades psychologists have been discussing how reflections display a reversal of the left-right orientation (Corballis, 2000; Gardner, 1964; Gregory, 1987, 1996; Haig, 1993; Ittelson, 1993; Ittelson, Mowafy, \& Magid, 1991; Morris, 1993; Navon, 1987; Tabata \& Okuda, 2000; Takano, 1998). Recently, it has been demonstrated experimentally that this reversal - defined in terms of the intrinsic frame of reference of an object or body - is not the only reversal characterizing the structure of reflections and neither is it the reversal which adult observers notice first: what they notice and describe is the opposite orientation of reflections along the axis which is orthogonal to the mirror with respect to an allocentric frame of reference (Bianchi \& Savardi, 2008; Savardi et al., 2010). It is also important to remember how mirrors are used in everyday life and in particular in relation to faces. People generally see their own face from a frontal view. As a consequence, selfrecognition has been found to be superior for full-frontal views as compared to other viewing angles, but this pattern does not extend to the recognition of other people's faces (Laeng \& Rouw, 2001; Troje \& Kersten, 1999).

These three types of correspondence and people's expectations concerning them are also often used in depicted scenes or manipulated in artworks that make use of mirrors, as exemplified by Bertamini, Latto et al. (2003); Hockney (2006) and Savardi and Bianchi (2014).

\subsection{Psychological models of the correspondence between material and reflected objects}

In terms of optics, there is a simple principle underlying reflections in a planar mirror. The ray of light forms incidence and reflection angles that are equal and coplanar, and the distances of the corresponding points along these rays are also equal. However, cognitive scientists have shown that even when adults have explicit knowledge of this law, they do not use it to predict the behaviour of reflections. This was revealed when participants in an experiment were asked to predict when a person walking parallel to a mirror hanging on a wall would start seeing his/her reflection or the reflection of another object (Croucher et al., 2002). The same occurred when, in another experiment, the participants were requested to predict the extension and angle of a mirror's field of view, given various positions of the observer (Bianchi \& Savardi, 2012). Beyond these two studies, which directly tested whether people possess an explicit knowledge of the physical rule of reflection even if they do not use it, one can in general maintain that all the errors reported in the literature on naïve optics are indirect proof that people do not apply the correct physical rule. If people were applying it, errors would be rare.

Psychologists have tried to understand how naïve subjects connect the reflected world with the material world. Some of the initial hypotheses, which resulted from the debate on the mirror question and the left-right mirror reversal (e.g. Corballis, 2000; Gregory, 1996; Tabata \& Okuda, 2000; Takano, 1998), called into play viewpoint reversals, representational reversals and optic reversals. Recently, on the basis of various different types of errors made by adults (including left-right reversal), two further hypotheses have been put forward. We refer to these as the "rotational geometry hypothesis" and the "vector geometry hypothesis".

1) Rotational geometry: People may think of the virtual world in a mirror in terms of a rotation of the world through the surface of the mirror. This was first suggested by a localization error discovered when participants in an experiment were asked to predict in which part of a mirror a person walking parallel to it would see their reflection appear. Around $20-40 \%$ expected it to appear at the farther rather than the nearer edge (Croucher et al., 2002). This is compatible with the idea that they expected the virtual world to be rotated $180^{\circ}$ with respect to the material world. However, when the participants were asked to identify the correct reflection from a series of pictures showing a room and its reflection in a mirror, they were able to say that the picture where the reflection showed a $180^{\circ}$ rotation of the room was incorrect (Croucher et al., 2002). In another study participants were asked to look at reflections of simple objects and describe the relationship between the reflection and the object. Participants almost never described the reflection as being rotated with respect to the object (Savardi et al., 2010, exps. 4-5). These results suggest that although some data is compatible with the idea that people think of reflections in terms of a rotation (Hecht, Bertamini, \& Gamer, 2005) this is not a heuristic that people consciously adopt. The rotational hypothesis has been re-proposed in a less radical version by Muelenz, Hecht, and Gamer (2010). They showed that the reconstruction of the virtual world is systematically rotated counter-clockwise by an average angle of two degrees. However, as pointed out by the authors, this rotational error accounts for small quantitative errors of localization but does not explain more blatant qualitative errors such as those previously mentioned.

2) Vector geometry: These more serious qualitative errors have led researchers to wonder whether the correspondence between material and reflected worlds is cognitively modelled in terms of identity and opposition (Bianchi \& Savardi, 2008, 2009; Savardi et al., 2010). This 
hypothesis emerged from the results of perceptual tasks that required adults to describe the reflections of their bodies, other people's bodies and simple objects and movements in plane mirrors (in various positions) with respect to the material objects/bodies (Bianchi \& Savardi, 2008, 2009; Savardi et al., 2010, exps. 4-5). Prediction tasks manifesting the generalization of the heuristics which state that "the mirror does the same" or that "the mirror does the opposite" (Savardi et al., 2010, studies 1-3; Bianchi \& Savardi, 2012) also led to similar conclusions. The geometry of reflections in plane mirrors can be thought of as a lawful combination of two components: one, parallel to the mirror and identical, and another orthogonal to the mirror and opposite (see Fig. 1).

3) When an object is positioned parallel to a mirror or orthogonal to it, one of the two components is null and the relation is defined by the other component: i.e. the orientation is identical in the first case (Fig. 1a) and opposite in the second case (Fig. 1b). When an object is positioned at an angle (Fig. 1c, d), both components interact and the result is a mixture of the two. Observers, however, often simplify these latter more complex conditions and apply the rule that holds for the simpler conditions (i.e. parallel and orthogonal), expecting the reflection to behave as shown in Fig. 2. These erroneous patterns reveal an incorrect generalization of, respectively, the rule that "the reflection does the same" (i.e. error type II, which means Identity in both components) which is what one sees when something moves parallel to a mirror (Fig. 1a), or the rule that "the reflection does the opposite" (i.e. error type 00, which means Opposition in both components) which is what one sees when something moves orthogonally to a mirror (Fig. 1b).

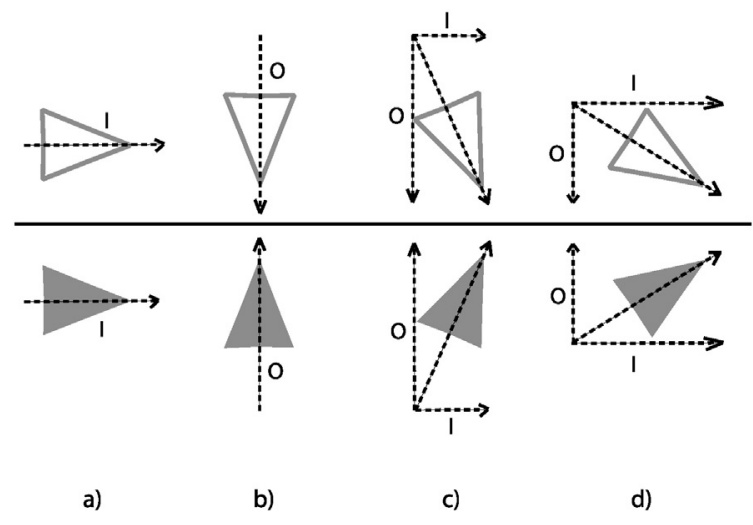

Fig. 1. Diagrams showing the relationship between the orientation of a moving object and that of its reflection in terms of two vector components: one parallel to the mirror and with the material movement and the reflection identical (I) and the other orthogonal to the mirror and with the material movement and its reflection opposite $(0)$. For more details see the main text.

\section{The ability to predict corresponding movements in reflections}

We know from the literature that when asked to predict the reflected movement corresponding to a simple straight motion (at various angles of incidence with respect to the mirror, from parallel to orthogonal), people make systematic mistakes (Savardi et al., 2010, exps. 1 and 3):

a) the errors relate to three types of incorrect patterns, as shown in Fig. 2;

b) incorrect responses are more frequent when a paper and pencil task is used as compared to when the prediction is evoked in a real-life setting (i.e. when the participants enter a room along pre-defined

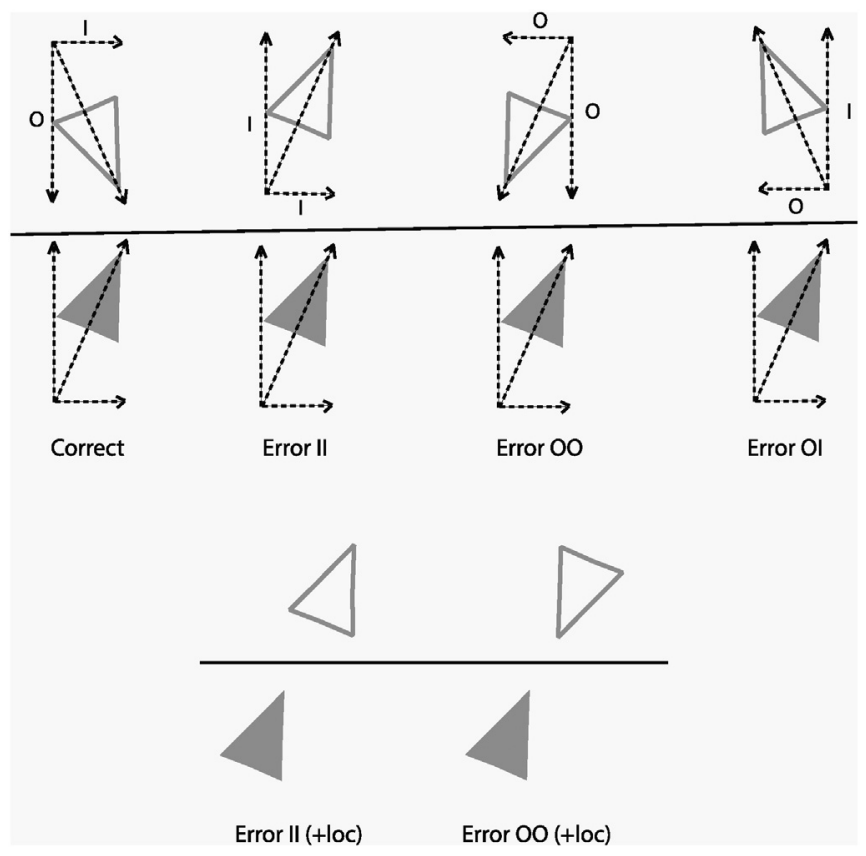

Fig. 2. Correct reflection and the pattern of errors defined according to vector geometry (as described in Fig. 1). These errors consist of a simplification of the geometry of reflections to mere identity (error type II: "the reflection does the same") and mere opposition (error type 00: "the reflection does the opposite") or involve confusion concerning which component is identical and which is opposite (error type OI). In error types II and $\mathrm{OO}$ the reflection is usually misplaced: it does not occupy the position it would in reality but is aligned along the main axis of the figure, as represented in the diagrams in the bottom row.

trajectories towards or away from a fake mirror);

c) in the real-life setting not only does the percentage of error decrease, but also only one of the two more frequent errors in the paper and pencil task (i.e. types II and OO) persists, i.e. the error manifesting the belief that "the reflection does the opposite" (OO).

These are the results from studies where participants were asked to predict reflections starting from material objects. The two experiments presented in this paper "reverse" the direction of the prediction since we wondered whether the previous findings would be confirmed when predictions were based on the reflection rather than the material object. We had no specific reason to expect the tasks to be asymmetrical. However, since it has been found that most people respond to these types of tasks by imagining a scenario rather than by applying geometrical or optical rules (see the reports of participants in Bianchi \& Savardi, 2012, or Yates et al., 1988; see also Bianchi \& Savardi, 2014), symmetry could not be taken for granted. Asymmetrical results would indicate that there is a cognitive difference between how people mentally model a reflected world from the corresponding material world and vice versa. This could lead to new insights into how people relate to mirrors and provide useful information on spatial modelling related to material and virtual worlds.

In the first study presented in this paper, naïve predictions were studied by means of paper and pencil tasks. This type of task is common in the previously cited literature on naïve optics and has also, more in general, frequently been found in literature on naïve physics since the original pioneering works by McCloskey et al. (1980). A handful of studies have involved asking participants to recognize the correct pattern rather than making a prediction of their own (e.g. for naïve physics: Bozzi, 1959; McAfee \& Proffitt, 1991; Runeson, 1974; for studies concerning mirrors: Croucher et al., 2002; Muelenz et al., 2010; Sareen, Ehinger, \& Wolfe, 2014) and people usually perform better in this type of study. This is evidence that recognizing as compared to 


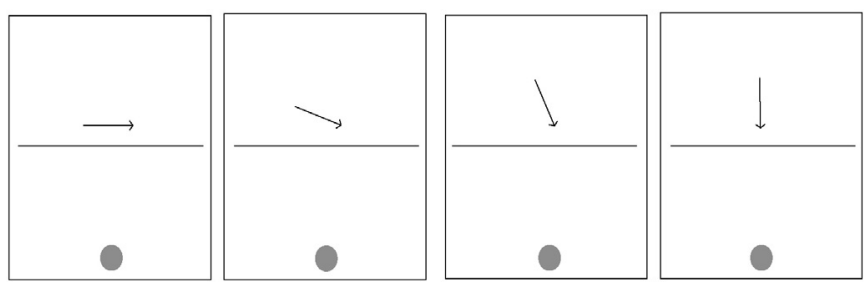

Fig. 3. The stimuli used in the paper and pencil task (from left to right: $0^{\circ}, 22^{\circ}, 67^{\circ}, 90^{\circ}$ ). Instructions: "The drawing represents an aerial view of a mirror (the longer line) and the movement of an object (indicated by the arrow) reflected in a mirror. You are standing where the grey circle is. Draw an arrow in front of the mirror indicating the movement that would give rise to that reflection."

producing a prediction (regarding a trajectory, a movement or an orientation) involves different cognitive processes. These may be related to memory, prototypical imagination, implicit knowledge or explicit knowledge and this is exactly what has stimulated twenty years of research in this area. As a result of this and due to the fact that it enables us to make direct comparisons with previous literature, we too decided to use paper and pencil tasks in our first experiment.

\section{Experiment 1}

The first experiment was designed to match Experiment 1 in Savardi et al. (2010). Participants were provided with paper diagrams (see Fig. 3) which were identical in size and appearance to that used in the original study, but they had been "inverted" with regard to the direction of the prediction: instead of telling participants that they were looking at drawings representing the movements of an object and asking them to predict the movement in the corresponding reflection, they were told that they were looking at reflections and that they had to predict how the corresponding material object would move in order to generate those reflections.

The aim of the study was to understand whether the number of errors and the types of error would vary in the two prediction conditions. We considered only movements towards the mirror (not away from the mirror) since no significant differences had emerged between the two conditions in the original study.

\subsection{Method}

\subsubsection{Participants}

Sixty undergraduate students (aged 20 to 25; 34 females) took part in the study. They were undergraduate students doing an introductory course in General Psychology at the University of Verona (i.e. the sample of participants was comparable to those who took part in the original study carried out by Savardi et al.). They were naïve with respect to the specific subject of the study.

\subsubsection{Procedure}

Each participant was presented with four sheets of paper, in random order, each showing a schematic drawing as seen from above (an aerial view) of a mirror with an arrow representing the movement reflected in the mirror. The movements were all linear movements at angles of $0^{\circ}$ (i.e. parallel), $22^{\circ}, 67^{\circ}$ and $90^{\circ}$ (i.e. orthogonal) with respect to the surface of the mirror (see Fig. 3). Participants were asked to draw an arrow indicating the movement of the material object that would correspond to the movement in the reflection. ${ }^{1}$ The instructions were given verbally

\footnotetext{
${ }^{1}$ In the original paper where the "reverse" task was used (Savardi et al. 2010), the arrows shown in Fig. 3 were printed on the opposite side with respect to the mirror. They represented the movement of the material object (not of the reflection as in our case) and were therefore located in the real space with the observer and not in the virtual space. The angles of inclination were the same. In the original study, both movements towards the mirror and away from the mirror were considered. Since no significant differences emerged between these two conditions, in the present study we considered only movements towards the mirror.
}

by the experimenter and were also printed at the top of each sheet. Before they started, the experimenter verified that the participants had understood the task and asked if they had questions.

\subsection{Results}

Responses were analysed in terms of the two components described in Fig. 1, one parallel and the other orthogonal to the mirror. In addition to correct responses, there were three types of errors, as represented in Fig. 2:

- Error type $O O$ (parallel component: Opposite; orthogonal component: Opposite).

- Error type II (parallel component: Identical; orthogonal component: Identical)

- Error type OI (parallel component: Opposite; orthogonal component: Identical).

Both Error type $\mathrm{OO}$ and Error type II are oversimplifications: in one case the reflection is expected to do the opposite $(\mathrm{OO})$ to the material object; in the other case, it is expected to do the same (II) as the material object. In Error type OI there is some confusion regarding the identity and opposition of the two components: they are inverted with respect to how they should be. Classifications were made by two independent raters (Cohen's kappa inter-rater agreement $=0.96$ ).

The responses from Experiment 1 (i.e. predictions of the movement of a material Object based on the reflection, from now on PredictO) were compared with the data relating to the inverse condition (i.e. predictions of a movement in a Reflection based on the material movement, from now on PredictR), i.e. the dataset from Experiment 1 in Savardi et al. (2010). The overall set of data was analysed with Generalized Mixed Effect Models (GLMMs). The problem with frequency data is that they may be correlated (e.g. when a number of predictions are made by the same participant, as is the case in our study since Angle was studied within subjects) and as a result may violate the independence assumption of both Chi square and log-linear analyses. Generalized Mixed Effects Models (GLMMs) are indicated in this case (Bates, Maechler, Bolker, \& Walker, 2014a, 2014b) since they allow us to treat various types of dependent variables, from binomial to scale values. GLMMs were thus used in the following analyses, with Angle $\left(0^{\circ}, 22^{\circ}, 67^{\circ}\right.$ and $\left.90^{\circ}\right)$ and Prediction condition (PredictO, PredictR) as fixed effects, and Subject as a random effect. Various dependent variables were considered, based on the specific research question being addressed.

\subsubsection{Correct versus incorrect responses}

We first analysed the number of participants who gave correct versus incorrect (independently of the type of error) answers. As shown in Table 1, the majority of participants were able to predict the correspondence between the movement of the material object and its reflection. However the percentage of errors ranged between $13.3 \%$ and $51.2 \%$ (this latter in the PredictR condition at $67^{\circ}$ ). If one considers that the movements were extremely simple, these percentages are rather high.

To understand whether participants found the two conditions (PredictO and PredictR) equally difficult and whether for some angles the prediction was more difficult than for others (as the data in Table 1 suggest), we carried out a first GLMM with correct/incorrect as a binary dependent variable (a logit link function was used), Angle and Condition as fixed effects and Subject as a random effect. Both the Angle of incidence $\left(\mathrm{F}_{(3,554)}=15.215, p<0.001\right)$ and the Prediction condition were significant $\left(\mathrm{F}_{(1,554)}=5.000, p=0.026\right)$, while no significant interaction emerged between the two effects $\left(F_{(3,554)}=0.598, p=0.617\right)$. Let us focus on the significant effect of Angle of incidence first: as shown in Fig. 4 (top row), correct responses were less frequent for intermediate angles $\left(22^{\circ}\right.$ and $\left.67^{\circ}\right)$ and more frequent when the geometry of identity and opposition is simpler since one of the two components is null, i.e. 
Table 1

Frequencies of correct responses and of the various types of errors for each of the stimuli presented in the two conditions: prediction of the material object based on the reflection (PredictO) and prediction of the reflection based on the material object (PredictR). Percentages are calculated by row, i.e. over the total number of responses collected for each of the stimuli (participants were in total 60 in the PredictO condition and 83 in the PredictR condition).

\begin{tabular}{|c|c|c|c|c|c|c|}
\hline \multirow[t]{2}{*}{ Condition } & \multirow{2}{*}{$\begin{array}{l}\text { Stimuli } \\
\text { (defined } \\
\text { by Angle) }\end{array}$} & \multicolumn{5}{|c|}{ Responses } \\
\hline & & Correct & $\begin{array}{l}\text { Error } \\
\text { II }\end{array}$ & $\begin{array}{l}\text { Error } \\
\text { OI }\end{array}$ & $\begin{array}{l}\text { Error } \\
\mathrm{OO}\end{array}$ & Total \\
\hline \multirow[t]{10}{*}{ PredictO } & $0^{\circ}$ & 51 & & 0 & 9 & 60 \\
\hline & & $85.0 \%$ & & $0.0 \%$ & $15.0 \%$ & $100.0 \%$ \\
\hline & $22^{\circ}$ & 40 & 10 & 4 & 5 & 59 \\
\hline & & $67.8 \%$ & $16.9 \%$ & $6.8 \%$ & $8.5 \%$ & $100.0 \%$ \\
\hline & $67^{\circ}$ & 36 & 11 & 2 & 9 & 58 \\
\hline & & $62.1 \%$ & $19.0 \%$ & $3.4 \%$ & $15.5 \%$ & $100.0 \%$ \\
\hline & $90^{\circ}$ & 52 & 8 & 0 & & 60 \\
\hline & & $86.7 \%$ & $13.3 \%$ & $0.0 \%$ & & $100.0 \%$ \\
\hline & Total & 179 & 29 & 6 & 23 & 237 \\
\hline & & $75.5 \%$ & $12.2 \%$ & $2.5 \%$ & $9.7 \%$ & $100.0 \%$ \\
\hline \multirow[t]{10}{*}{ PredictR } & $0^{\circ}$ & 63 & & 0 & 19 & 82 \\
\hline & & $76.8 \%$ & & $0.0 \%$ & $23.2 \%$ & $100.0 \%$ \\
\hline & $22^{\circ}$ & 49 & 14 & 5 & 12 & 80 \\
\hline & & $61.3 \%$ & $17.5 \%$ & $6.3 \%$ & $15.0 \%$ & $100.0 \%$ \\
\hline & $67^{\circ}$ & 40 & 13 & 3 & 26 & 82 \\
\hline & & $48.8 \%$ & $15.9 \%$ & $3.7 \%$ & $31.7 \%$ & $100.0 \%$ \\
\hline & 90 & 72 & 11 & 0 & & 83 \\
\hline & & $86.7 \%$ & $13.3 \%$ & $0.0 \%$ & & $100.0 \%$ \\
\hline & Total & 224 & 38 & 8 & 57 & 327 \\
\hline & & $68.5 \%$ & $11.6 \%$ & $2.4 \%$ & $17.4 \%$ & $100.0 \%$ \\
\hline
\end{tabular}

at $0^{\circ}$ and $90^{\circ}$. In this latter case, correct responses ranged between $76.8 \%$ and $86.7 \%$ of the total number of responses. Bear in mind that in a GLMM model like the one used here (which works on a logit transformation of a binary variable), an equal proportion of the two categories (correct/incorrect) corresponds to a logit value of 0.5 , whereas logit values greater than 0.5 indicate, in our case, that correct $>$ incorrect responses and values lower than 0.5 indicate that correct < incorrect responses. Thus, as shown in Fig. 4 , at $22^{\circ}$ and $67^{\circ}$ responses were still more frequently correct than incorrect, but the estimated means were not far from 0.5 (i.e. an equal proportion of correct and incorrect responses). This trend was common to both prediction conditions (PredictO, PredictR), as the absence of a significant interaction between Angle and Prediction condition confirmed. However, as the main effect of Prediction revealed (bottom diagram in Fig. 4), participants generally made more correct predictions when asked to start from the reflection and predict the material movement (PredictO) than vice versa (PredictR).

\subsubsection{Types of error}

Which specific trajectory was predicted when participants gave incorrect responses? Some initial descriptive observations can be made based on the overall percentages shown in Table 1. OI type errors were not frequent: in both prediction conditions they ranged from 3\% to $7 \%$ of the total number of responses. Type II errors ranged from $13 \%$ to $19 \%$ in both prediction conditions (Predict0: $13.3 \%-19.0 \%$; PredictR: 13.3\%-17.5\%). Type 00 errors were overall more frequent when participants predicted the reflection starting from the material movement (PredictR: 15\%-31\%) than when predicting the material movement starting from the reflection (Predict0: 8.5\%-15.5\%). We need to remember that, in contrast to type OI errors that manifest a confusion between which component is opposite and which is identical in the reflection, error types II and $\mathrm{OO}$ display a reduction in the geometry of the reflection to only one of the two components: error type II demonstrates the expectation that a mirror will do the same - which is true for movements which are parallel to the mirror or at $0^{\circ}$ - and error type 00 demonstrates the expectation that a mirror will do the opposite - which is true for movements which are orthogonal to the mirror or at $90^{\circ}$. In neither of these cases (error types II and 00) do people expect a reflection to behave in the same way and in the opposite way. Therefore, the frequencies of the three types of errors suggest that the most frequent errors involve simplified predictions, i.e. that the mirror will do the same (error type II) or the opposite (error type 00). This is in agreement with what Savardi et al. (2010) found. The same goes for the trend relating to error type $\mathrm{OO}$ which becomes more frequent as the direction of the
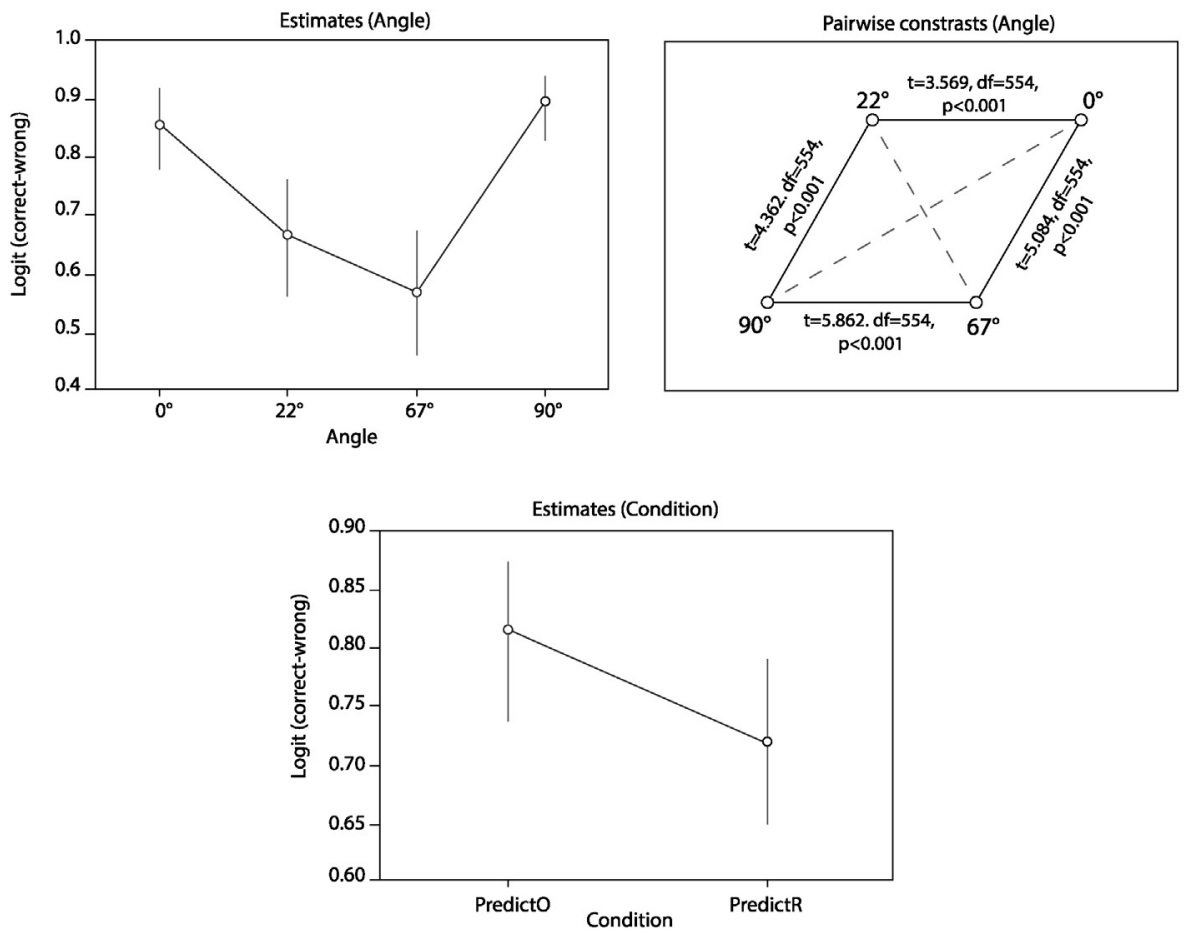

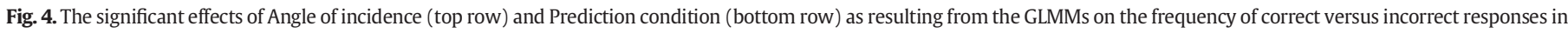

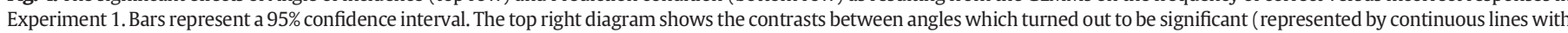
corresponding statistics) and which did not (represented by dashed lines). 
movement gets closer to $90^{\circ}$ (i.e. where the movement in the reflection and that of the material object are in effect opposite).

We focused on the two more frequent categories of incorrect responses (i.e. error types II and OO) and we wondered whether these two types of errors affected the two prediction conditions in different ways. Since it is statistically appropriate to parameterize a multinomial model as a series of binomial contrasts (Allison, 1984; Dobson \& Barnett, 2008), we carried out a second GLMM on the frequency of the two categories of response (error type II and error type OO), with Subject as a random effect and Angle and Prediction condition as fixed effects (and using a binomial logit link function).

A significant effect of the Prediction condition emerged $\left(\mathrm{F}_{(1,143)}=\right.$ $8.290, p<0.005$ ): error type 00 was significantly more frequent when participants were given the material movement and asked to predict the reflected movement (PredictR), than in the inverse condition (PredictO), $\mathrm{t}=3.047, p=0.003$. Fig. 5 represents this main effect. Bearing in mind that a logit value of 0.5 indicates an equal proportion of the two categories of the binary variable (i.e. error type $\mathrm{OO}=$ error type II) and that error type II is used as a reference category, Fig. 5 not only shows that error type 00 was more frequent in PredictR than in PredictO, but also that when participants were asked to predict the reflection (PredictR), error type 00 was more frequent than error type II (the mean of the effect is 0.686 , i.e. $>0.5$ ). Conversely, when participants were asked to predict the material movement starting from the reflection (PredictO), error type 00 was less frequent than type II (the mean of the effect is 0.348 , i.e. $<0.5$ ).

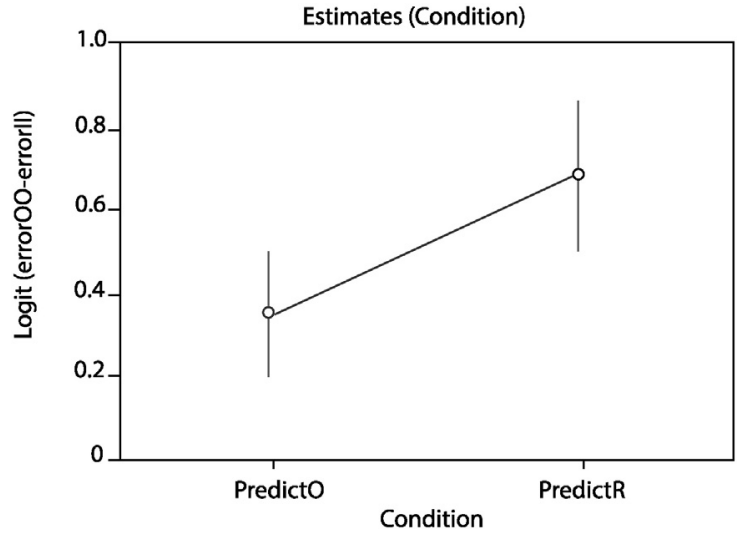

Fig. 5. The significant effect of the Prediction condition which resulted from the GLMM on the frequency of error type 00 and error type II in Experiment 1. Bars represent a 95\% confidence interval.

\section{Experiment 2}

The results from Experiment 1 suggest that the two tasks (predict the reflection and predict the material movement) are not identical and that some asymmetries emerge, at least when paper and pencil tasks are used. Previous studies on naïve physics and optics have demonstrated that with real-life settings results might significantly change. But this is not necessarily the rule. For instance, the errors concerning the field of view of reflections described in the introduction (Bertamini et al., 2010; Bianchi \& Savardi, 2012; Croucher et al., 2002) also arose in real-life settings. Experiment 2 used locations within a room instead of a paper and pencil task. Just as in Experiment 1, in Experiment 2 we used linear movements at various angles of incidence with respect to the mirror and two prediction conditions.

\subsection{Method}

\subsubsection{Participants}

Two groups of undergraduate students (ages ranging from 20 to 38) took part in the experiment. Forty-two ( 24 females) participated in the "predict the material movement" condition (PredictO); forty (28 females) participated in the "predict the reflection" condition (PredictR).

\subsubsection{Procedure}

A two metre long strip of white adhesive tape was set on the floor of an empty room. Participants were told to imagine a mirror positioned vertically along this strip.

\subsubsection{Condition PredictO}

Four paths were marked on the floor with transparent tape on the opposite ("reflection") side of the pretend mirror with respect to the participant. These paths were at various angles of incidence: $0^{\circ}$ (parallel to the mirror), $90^{\circ}$ (orthogonal to the mirror), $22^{\circ}$ and $67^{\circ}$. The experimenter moved along these paths, one at a time (with the order being randomized between participants), towards the fake mirror. Participants were told that the experimenter was playing the role of the reflection. They were invited to watch the movement being performed and then do what they believed would be the material movement that corresponded to what they had just seen. At the beginning of the experiment, and before each movement was performed by the experimenter, participants were asked to stay to the side of the fake mirror rather than directly in front of it. This was done so that as part of the task they had to make a decision about the starting point as well as the trajectory of the movement they would subsequently make.

\subsubsection{Condition PredictR}

The procedure was similar to the previous condition except that this time the strips of adhesive tape indicating the 4 paths were set on the floor on the same side as the participant. The experimenter invited each participant to walk along the paths, one at a time, towards the mirror (with the order of the paths again being randomized between participants). They were then asked to indicate to the experimenter playing the role of the reflection how he/she should move in order to simulate the corresponding movement in the reflection. In order to avoid conditioning the participant's decision concerning the starting point of the reflection, at the beginning of the experiment and before each movement was performed by the subject the experimenter stood to the side of the pretend mirror. ${ }^{2}$

\subsection{Results}

\subsubsection{Correct versus incorrect responses}

Did the number of correct responses vary based on the Angle of movement and the Prediction condition? To answer this question a GLMM was used, with correct/incorrect as the binary dependent variable (logit link function), Angle $\left(0^{\circ}, 22^{\circ}, 67^{\circ}, 90^{\circ}\right)$ and Condition (PredictO, PredictR) as fixed effects, and Subject as a random effect. The analysis revealed a significant effect of Angle $\left(\mathrm{F}_{(3,318)}=11.469, p<0.001\right)$ but no effect of the Prediction condition $\left(\mathrm{F}_{(1,318)}=0.211, p=0.64\right)$. Neither was there any interaction between Angle and Condition $\left(\mathrm{F}_{(3,318)}=1.768, p=0.153\right)$. As shown in Fig. 6, correct responses were particularly frequent at $0^{\circ}$ and $90^{\circ}$, with no significant difference between the two, whereas correct responses were significantly less frequent at $22^{\circ}$ and $67^{\circ}$. This trend was confirmed both when participants were asked to predict the reflection (PredictR) and when they were asked to predict the material object (PredictO), as the absence of interaction between Angle and Condition indicated. The trend is similar to that which emerged in Experiment 1 confirming that predicting is easier (or at least more successful) in the

\footnotetext{
2 This procedure is a simplification of that used in Savardi et al. (2010).
} 

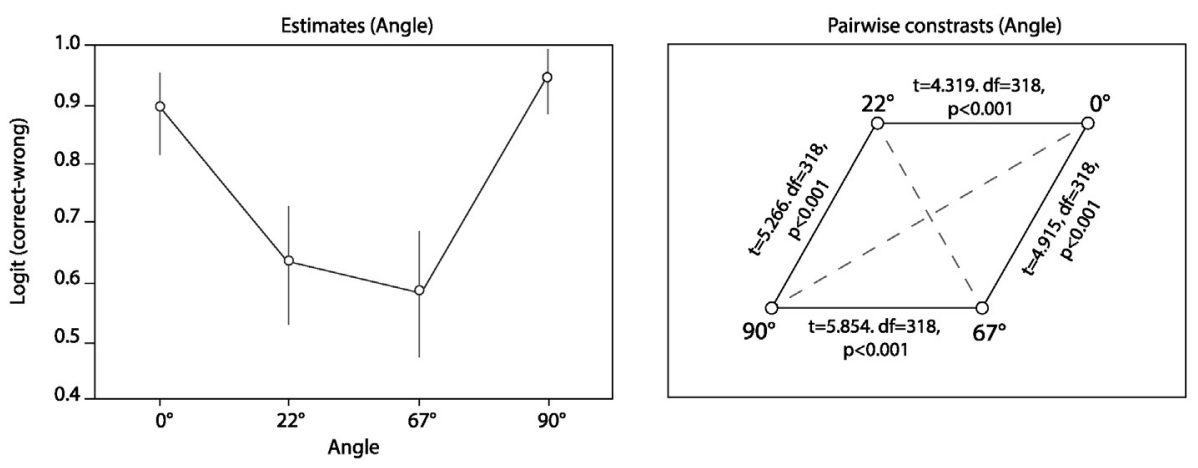

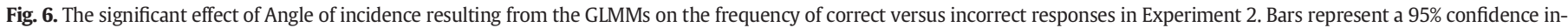

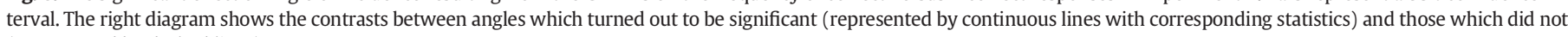
(represented by dashed lines).

two cases where the geometry of reflection is simplified because it only involves the identity component (movement parallel to the mirror, or $0^{\circ}$ ) or the opposition component (movement orthogonal to the mirror, or $90^{\circ}$ ). In this real-life setting there were no differences between basing the prediction on the reflection (PredictO) or on the real movement (PredictR), at least in terms of the frequency of correct/incorrect responses. As we will see in a moment, however, asymmetries did emerge concerning the various types of errors.

A second GLMM was conducted to study whether distribution of correct and incorrect responses varied between the two experiments - with correct/incorrect as the binary dependent variable (logit link function), Angle $\left(0^{\circ}, 22^{\circ}, 67^{\circ}, 90^{\circ}\right)$, Condition (PredictO, PredictR) and Task (paper, real-life) as fixed effects, and Subject as a random effect. Only two effects emerged: Angle $\left(\mathrm{F}_{(3,875)}=24.608, p<0.001\right)$, and Task $\left(F_{(1,875)}=7.927, p=0.005\right)$. The latter indicates that performance was better in the real-life condition (Experiment 2). The main effect of Angle (and the absence of interaction with the other variables) confirmed the overall effect which we had already noticed in the separate analyses for each of the two experiments: there was a higher number of correct predictions for movements which were parallel or orthogonal to the mirror $(p<0.001)$ than for movements at intermediate angles.

\subsubsection{Types of error}

In this section we look at the various types of incorrect predictions made by participants. The data in Table 2, as compared to

Table 2

Frequencies of correct predictions and of the various types of errors in Experiment 2. Percentages are calculated by row, i.e. they pertain to the total number of responses collected for each stimulus.

\begin{tabular}{|c|c|c|c|c|c|}
\hline \multirow[t]{2}{*}{ Condition } & \multirow{2}{*}{$\begin{array}{l}\text { Stimuli } \\
\text { (defined by Angle) }\end{array}$} & \multicolumn{4}{|c|}{ Responses } \\
\hline & & Correct & Error II & Error OO & Total \\
\hline \multirow[t]{10}{*}{ PredictO } & $0^{\circ}$ & 37 & & 5 & 42 \\
\hline & & $88.1 \%$ & & $11.9 \%$ & $100.0 \%$ \\
\hline & $22^{\circ}$ & 27 & 3 & 12 & 42 \\
\hline & & $64.3 \%$ & $7.1 \%$ & $28.6 \%$ & $100.0 \%$ \\
\hline & $67^{\circ}$ & 29 & 1 & 12 & 42 \\
\hline & & $69.0 \%$ & $2.4 \%$ & $28.6 \%$ & $100.0 \%$ \\
\hline & $90^{\circ}$ & 38 & 4 & & 42 \\
\hline & & $90.5 \%$ & $9.5 \%$ & & $100.0 \%$ \\
\hline & Total & 131 & 8 & 29 & 168 \\
\hline & & $78.0 \%$ & $4.8 \%$ & $17.3 \%$ & $100.0 \%$ \\
\hline \multirow[t]{10}{*}{ PredictR } & $0^{\circ}$ & 37 & & 3 & 40 \\
\hline & & $92.5 \%$ & & $7.5 \%$ & $100.0 \%$ \\
\hline & $22^{\circ}$ & 24 & 0 & 15 & 39 \\
\hline & & $61.5 \%$ & $0.0 \%$ & $38.5 \%$ & $100.0 \%$ \\
\hline & $67^{\circ}$ & 18 & 0 & 21 & 39 \\
\hline & & $46.2 \%$ & $0.0 \%$ & $53.8 \%$ & $100.0 \%$ \\
\hline & $90^{\circ}$ & 40 & 0 & & 40 \\
\hline & & $100.0 \%$ & $0.0 \%$ & & $100.0 \%$ \\
\hline & Total & 119 & 0 & 39 & 158 \\
\hline & & $75.3 \%$ & $0.0 \%$ & $24.7 \%$ & $100.0 \%$ \\
\hline
\end{tabular}

those reported in Table 1 (concerning Experiment 1), suggest some initial considerations. In a real-life setting: i) error type OI disappeared and ii) error type II became less frequent. In the paper and pencil task (Experiment 1), 13\%-17.5\% of the total of responses were error type II, whereas in the real-life setting (Experiment 2 ) these only amounted to $2.4 \%-9.5 \%$; iii) error type 00 was more robust and persistent - in the real-life setting (Experiment 2) it represented $12 \%-29 \%$ of the total number of responses when participants were asked to predict the material movement based on the reflection (Predict0). This went up to 54\% when participants were asked to predict the reflection (PredictR).

To better analyse responses, a GLMM was carried out on the frequency of the two types of errors (II and OO) with Subject as random effect, Angle and Prediction condition as fixed effects (we used a binomial logit link function). The analysis confirmed the significant effect of the Prediction condition $\left(\mathrm{F}_{(1,74)}=4.634, p=0.035\right)$ : as in Experiment 1, error type $\mathrm{OO}$ was significantly more frequent when participants were asked to predict the reflection (PredictR) than when they were asked to predict the material movement from the reflection (PredictO), $\mathrm{t}=$ $2.580, p=0.012$. As Fig. 7 makes clear, not only is there an asymmetry between the two conditions, but also the estimated means (in logit values) are greater in both conditions than 0.5 (Mean (Predicto) $=$ $0.783, \mathrm{SE}=0.068 ;$ Mean $_{\text {(PredictR })}=0.971, \mathrm{SE}=0.027$ ), i.e. error type $\mathrm{OO}$ was more frequent than error type II.

A final GLMM was conducted to study the effect of the task (paper and pencil versus real-life setting) on the distribution of error types II

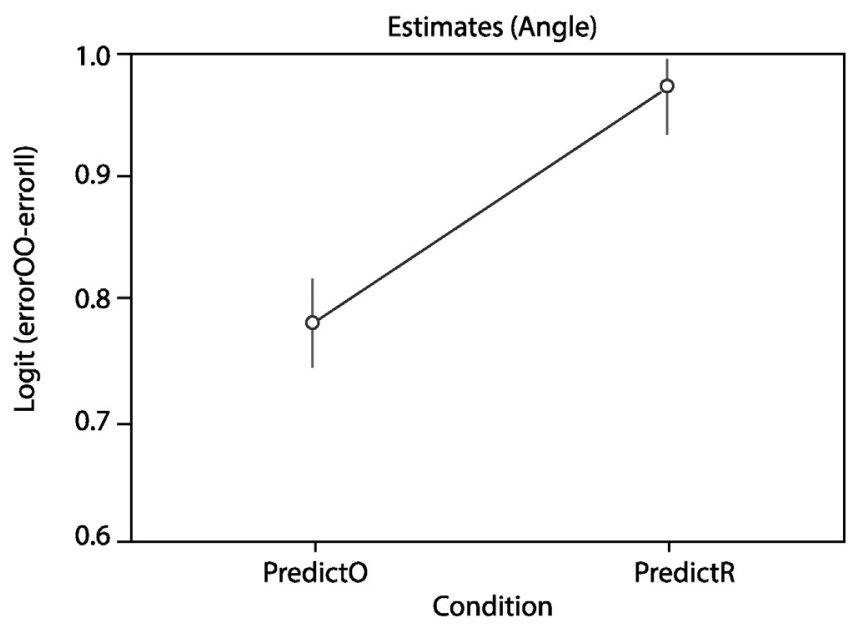

Fig. 7. Significant effect of the Prediction condition that resulted from the GLMMs on the frequency of error type $\mathrm{OO}$ and error type II in Experiment 2 (see text; error type II is the reference category). Bars represent a 95\% confidence interval. 

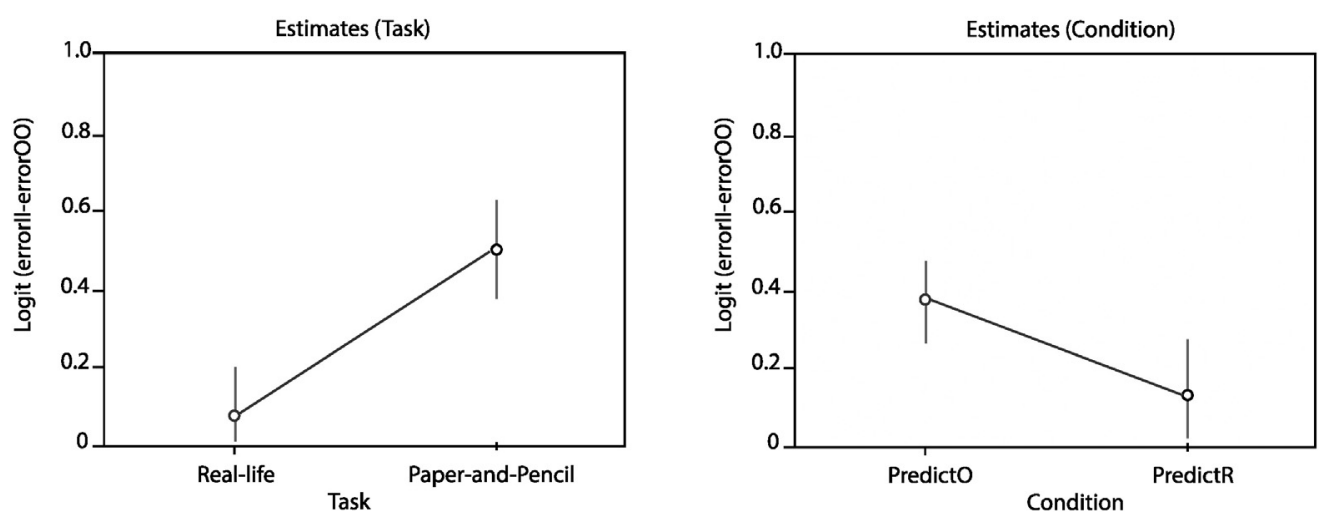

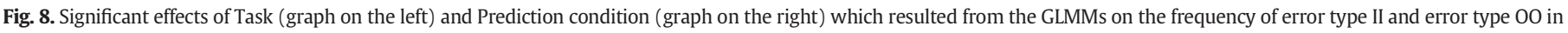
Experiment 1 (paper and pencil task) and Experiment 2 (real-life setting). Error type 00 is the reference category. Bars represent a 95\% confidence interval.

and 00. Since in the previous analysis only the Prediction condition (and not the Angle) turned out to affect the distribution of error types II and $\mathrm{OO}$, we included in the model only two fixed effects (Prediction condition and Task) in addition to Subject (as a random effect). ${ }^{3}$ Both Task $\left(\mathrm{F}_{(1,218)}=17.012, p<0.001\right)$ and Prediction condition $\left(\mathrm{F}_{(1,218)}=\right.$ $11.502, p=0.001$ ) turned out to be significant (see Fig. 8). Error type II was more frequent in the paper and pencil task (Experiment 1) as compared to the real-life task (Experiment 2), $\mathrm{t}=4.125, p<.001$, and when participants were asked to predict the material movement starting from the reflection (PredictO) rather than vice versa $(\mathrm{t}=$ $3.391, p<0.001)$.

\section{Final discussion}

In the Introduction, we discussed the issue of the correspondence between material objects/movements and reflections from a physical point of view but also in terms of how people perceive and understand this correspondence. As pointed out in the revised literature, people do not necessarily think of physical phenomena (and, in particular, the behaviour of mirror reflections) in terms of optical-geometrical rules: in most cases they base their predictions on the mental image they have of the scenario. Hence the fundamental question regards whether it is the same to move mentally from the material world to the virtual world as it is to go from the virtual to the material world. The results of the two studies can be summarised as follows.

\subsection{Experiment 1 (paper and pencil task)}

a) Performance was better when participants had to predict a movement starting from the reflection (PredictO) rather than predict the motion in the reflection starting from the material movement (PredictR). This asymmetry suggests that, even in the most abstract task, the participants did not approach it by merely drawing "construction lines" and applying a learned rule. If so, it would have made no difference which base they started from.

b) Despite the fact that the movement was simple, on average $24.5 \%$ of responses were incorrect in the condition that turned out to be the simplest (PredictO) and 31.5\% in the other condition (PredictR). For oblique movements with respect to the mirror, wrong predictions characterized up to $52 \%$ of responses.

c) The incorrect predictions manifested the use of a simplified geometry, i.e. the belief that "the mirror does the same" (error type II) or "the mirror does the opposite" (error type 00), rather than a

\footnotetext{
${ }^{3}$ A preliminary comparison of this model and one which also included the interaction between Prediction condition and Task revealed that the fit of the model did not improve when the interaction was included. For this reason and following the indication of the smaller AIC value, the interaction was not included.
}

confusion between which component does the same and which does the opposite (error type OI). This is also consistent with the fact that participants performed significantly better for movements which were parallel $\left(0^{\circ}\right)$ or orthogonal $\left(90^{\circ}\right)$ to the mirror, that is, when the movement and its reflection are in effect "the same" (at $0^{\circ}$ ) and when the movement and its reflection are in effect "opposite" (at $90^{\circ}$ ).

d) An asymmetry between the two conditions (PredictO and PredictR) emerged, not only in terms of the number of wrong predictions (as discussed in point a) but also in terms of the type of error. Error type $\mathrm{OO}$ was more frequent when participants had to predict the reflection (PredictR) than to predict the material movement (PredictO). This suggests that it is specifically when participants start from a material movement and are asked to determine what the corresponding reflection would be that they remember that reflections reverse something (i.e. "do the opposite") and apply this rule. Indeed, error type 00 eliminates the component of identity that a true reflection would exhibit (see Figs. 1 and 2).

This latter asymmetry concerning the type of error is a robust finding which also emerged also in a real-life setting (see points $g$ and $h$ ).

\subsection{Experiment 2 (real-life setting)}

e) Participants made fewer mistakes in the real-life condition as compared to the paper and pencil task. However, there were incorrect predictions in an average of $22 \%-25 \%$ of cases.

f) Performance was similar in both directions. Participants did not find it more difficult when they were asked to predict the reflection based on the material movement as compared to when they were asked to predict the material movement based on the reflection. In this sense there was no asymmetry in terms of the number of correct responses, but an asymmetry still remained in terms of the types of errors made (see points $g$ and $h$ ).

g) In the real-life condition, error type 00 became even more frequent than in the paper and pencil task, while the other two types of errors never (error type OI) or rarely (error type II) occurred.

h) As in Experiment 1, error type 00 was more frequent when people were asked to predict the reflection based on the material movement (PredictR) than in the reverse condition (PredictO).

With respect to the three elements of correspondence mentioned in the introduction, i.e. correspondence of size, correspondence of location and correspondence of orientation, our findings add new information concerning the last element (correspondence of orientation). They strengthen the hypothesis that people think of the correspondence 
between virtual and material worlds in terms of a vector geometry based on two heuristics: "the reflection does the same" and "the reflection does the opposite" (described in Section 1.2). The results of our experiments demonstrate the robustness of the latter in particular. This applies not only to paper and pencil tasks, but also to real-life simulations. This is consistent with (and thus consolidates) a previous finding in the experiment discussed in Savardi et al. (2010) which involved one of the two prediction conditions in the present study, that is, predicting the corresponding reflection based on a material movement (PredictR). However, in the present study we focused on the match with the inverse prediction condition (i.e. PredictO) in order to understand the heuristics and the limits of their application better. The differences which emerged between the incidence of error type 00 and error type II in the two tasks (PredictO, PredictR) in both Experiment 1 and Experiment 2 suggest that the way in which people think of reflections is not independent of the starting point (the "real world" or the "virtual world"). The expectation that the reflection of something will be opposite is particularly salient when people imagine how a reflection would be with respect to the real world rather than when they imagine how the real world would be with respect to a reflection. In other words, people tend to expect reflections to be opposite to the real world rather than vice versa. And indeed people sometimes expect the real world to be identical to the reflection (error type II), whereas they less frequently expect a reflection to be identical to the real world.

The results of our study constitute evidence of a cognitive asymmetry between reflections and their counterparts. This adds to Sareen et al. (2014) concerning the reality/unreality of reflections and their corresponding material objects. Their experiment involved a free-viewing labelling task with pictures showing ecological indoor scenes including a mirror. The results showed that people label reflected objects less frequently than their material counterparts. They also used a changedetection task and found that the disappearance of an object in a mirror was less easily detected than the disappearance of an object elsewhere in the room. It has been said that this is evidence that people treat the parts of images that represent reflections as somewhat less "real" than the parts that represent material objects/spaces (Sareen et al., 2014).

The asymmetry which emerged from our study has more to do with the geometry underlying the processes involved when the virtual world is mentally derived from the material one and vice versa. We hope that these findings will contribute towards future models regarding spatial reasoning about material objects or movements and their reflections. In particular our results specifically demonstrate that the components of identity and opposition which typically characterize how humans think of the relationship between material objects/movements and their reflection have different weights.

The movements considered in our experiments were very simple, straightforward movements. Despite this, errors emerged even in the real-life condition. Percentages of incorrect predictions were low (around 10\%) for orthogonal and parallel movements, but higher for intermediate angles where they varied between $31 \%$ and 54\%. We might wonder about the implications of all this when we use mirrors in situations where we have to control actions based on visual information. For instance when driving, the use of rear mirrors or mirrors placed at junctions and crossroads presupposes the capacity to predict where and in which direction a car is moving based on the movement seen in the reflection. The errors that emerged in the experiments presented in this paper suggest that it is worth studying people's ability to use mirrors in all situations when driving not only when making distance judgments (the latter has been addressed for example by Hahnel \& Hecht, 2012; Hecht \& Brauer, 2007; Higashiyama, Yokoyama, \& Shimono, 2001 and Higashiyama \& Shimono, 2004). The effect of training may be another important factor to consider. One might expect people to be very good at predicting movements based on a reflection in a mirror in familiar everyday situations such as driving (even though they make errors in more abstract lab tasks). However, neither rear view mirrors nor the mirrors at junctions and crossroads are flat. In this case, predictions become even more complex than those studied in this paper since predicting where a car is and how is it moving requires one to take into additional consideration the curvature of the mirror surface. And driving is only one example of an everyday activity which relies on an ability to use reflections to predict the real directions of motion.

\section{References}

Allison, P. D. (1984). Event history analysis. Newbury Park, CA: Sage Publications.

Bates, D., Maechler, M., Bolker, B., \& Walker, S. (2014a). lme4: Linear mixed-effects models using Eigen and S4 (R package version 1.1-7). (Retrieved from http://CRAN.R-project. org $/$ package $=\operatorname{lme} 4$ )

Bates, D., Maechler, M., Bolker, B., \& Walker, S. (2014b). lme4: Linear mixed-effects models using Eigen and S4. ArXiv e-print; submitted to Journal of Statistical Software. Retrieved from http://arxiv.org/abs/1406.5823.

Bertamini, M., Latto, R., \& Spooner, A. (2003a). The Venus effect: People's understanding of mirror reflections in paintings. Perception, 32, 593-599. http://dx.doi.org/10 1068/p3418.

Bertamini, M., Lawson, R., Jones, L., \& Winters, M. (2010). The Venus effect in real life and in photographs. Attention Perception E Psychophysics, 72, 1948-1964. http://dx.doi. org/10.3758/APP.72.7.1948.

Bertamini, M., \& Parks, T. E. (2005). On what people know about images on mirrors. Cognition, 98, 85-104. http://dx.doi.org/10.1016/j.cognition.2004.11.002.

Bertamini, M., Spooner, A., \& Hecht, H. (2003b). Naïve optics: Predicting and perceiving reflections in mirrors. Journal of Experimental Psychology: Human Perception and Performance, 29(5), 982-1002. http://dx.doi.org/10.1037/0096-1523.29.5.982.

Bianchi, I., \& Savardi, U. (2014). Grounding naive physics and optics in perception. The Baltic international yearbook for cognition logic and communication. Perception and concepts, vol. 6, http://dx.doi.org/10.4148/biyclc.v9i0.1081.

Bianchi, I., \& Savardi, U. (2012). What fits in into a mirror: Naïve beliefs on the field of view of mirrors. Journal of Experimental Psychology: Human Perception and Performance, 38(5), 1144-1158. http://dx.doi.org/10.1037/a0027035.

Bianchi, I., \& Savardi, U. (2009). Contrariety in plane mirror reflections. In U. Savardi (Ed.), The perception and cognition of contraries (pp. 113-128). Milan, Italy: Mc-Graw Hill.

Bianchi, I., \& Savardi, U. (2008). The relationship perceived between the real body and the mirror image. Perception, 5, 666-687.

Bianchi, I., Savardi, U., \& Bertamini, M. (2008). Estimation and representation of head size (People overestimate the size of their head - Evidence starting from the 15th century). British Journal of Psychology, 99(4), 513-531. http://dx.doi.org/10.1348/ $000712608 \times 304469$.

Bozzi, P. (1959). Le condizioni del movimento 'naturale' lungo i piani inclinati'. Rivista di Psicologia, LIII (II), 337-352 (English Translation by P. Bressan and P. Gaudiano 1989. "The Conditions for 'Natural' Motion Along Inclined Planes". Department of Psychology, UALR, 2801 South University, Little Rock, AR 72204, USA).

Corballis, M. C. (2000). Much ado about mirrors. Psychonomic Bulletin and Review, 7(1), 163-169.

Croucher, C. J., Bertamini, M., \& Hecht, H. (2002). Naïve optics: Understanding the geometry of mirror reflections. Journal of Experimental Psychology: Human Perception and Performance, 28, 546-562. http://dx.doi.org/10.1037//0096-1523.28.3.546.

Dobson, A. J., \& Barnett, A. (2008). An introduction to generalized linear models (Third edition ). Chapman \& Hall/CRC Texts in Statistical Science.

Gardner, M. (1964). The ambidextrous universe. New York: Basic Books.

Gregory, R.L. (1987). Mirror reversals, in Gregory R.L. (Eds): The Oxford companion to the mind, 491-493. Oxford: Oxford University Press.

Gregory, R. L. (1996). Mirrors in mind. New York: Freeman Spektrum.

Hahnel, U. J. J., \& Hecht, H. (2012). The impact of rear-view mirror distance and curvature on judgements relevant to road safety. Ergonomics, 55(1), 23-36. http://dx.doi.org/ $10.1080 / 00140139.2011 .638402$.

Haig, N. D. (1993). Reflections on inversion and reversion. Perception, 22, 863-868. http:// dx.doi.org/10.1068/p220863.

Hecht, H., \& Brauer, J. (2007). Convex rear view mirrors compromise distance and timeto-contact judgements. Ergonomics, 50(4), 601-614. http://dx.doi.org/10.1080/ 00140130601154954.

Hecht, H., Bertamini, M., \& Gamer, M. (2005). Naive optics: Acting on mirror reflections Journal of Experimental Psychology: Human Perception and Performance, 31(5), 1023-1038. http://dx.doi.org/10.1037/0096-1523.31.5.1023.

Higashiyama, A. (2004). Mirror vision: Perceived size and perceived distance of virtual images. Perception \& Psychophysics, 66, 679-691. http://dx.doi.org/10.3758/ BF03194911.

Higashiyama, A., \& Shimono, K. (2004). Mirror vision: Perceived size and perceived distance of virtual images. Perception \& Psychophysics, 66, 679-691. http://dx.doi.org/ 10.3758/BF03194911.

Higashiyama, A., Shimono, K., \& Zaitsu, W. (2005). Contraction of perceived size and perceived depth in mirrors. Psicológica, 26, 81-95.

Higashiyama, A., Yokoyama, Y., \& Shimono, K. (2001). Perceived distance of targets in convex mirrors. Japanese Psychological Research, 43, 13-24. http://dx.doi.org/10. 1111/1468-5884.00155.

Hockney, D. (2006). Secret knowledge. Rediscovering the lost techniques of the old masters. NY, USA: Viking Studio (Penguin Group).

Ittelson, W. H., Mowafy, L., \& Magid, D. (1991). The perception of mirror rotated objects. Perception, 20, 567-584.

Ittelson, W. H. (1993). Mirror reversals: Real and perceived. Perception, 22, 855-861. 
Jones, L., \& Bertamini, M. (2007). Through the looking glass: How the relationship between an object and its reflection affects the perception of distance and size. Perception, 36, 1572-1594. http://dx.doi.org/10.1068/p5605.

Lawson, R., \& Bertamini, M. (2006). Errors in judging information about reflections on mirrors. Perception, 35, 1265-1288. http://dx.doi.org/10.1068/p5498.

Lawson, R., Bertamini, M., \& Liu, D. (2007). Overestimation of the projected size of objects on the surface of mirrors and windows. Journal of Experimental Psychology: Human Perception and Performance, 33, 1027-1044. http://dx.doi.org/10.1037/0096-1523. 33.5.1027.

Laeng, B., \& Rouw, R. (2001). Canonical views of faces and the cerebral hemispheres. Laterality, 6, 193-224. http://dx.doi.org/10.1080/13576500042000115.

McAfee, E. A., \& Proffitt, D. R. (1991). Understanding the surface orientation of liquids. Cognitive Psychology, 23(3), 483-514.

McCloskey, M. (1983a). Intuitive physics. Scientific American, 248, 122-130.

McCloskey, M. (1983b). Naive theories of motion. In D. Gentner, \& A. Stevens (Eds.), Mental models (pp. 299-324). Hillsdale, NJ: Erlbaum.

McCloskey, M., Caramazza, A., \& Green, B. (1980). Curvilinear motion in the absence of external forces: Naive beliefs about the motion of objects. Science, 210(4474), 1139-1141.

McCloskey, M., Washburn, A., \& Felch, L. (1983). Intuitive physics: The straight-down belief and its origin. Journal of Experimental Psychology: Learning, Memory, and Cognition, 9(4), 636-649.

Melchior-Bonnet, S. (2001). The mirror. London: Routledge.

Morris, R. C. (1993). Mirror image reversals: Is what we see what we present? Perception, 22, 869-876.

Muelenz, C., Hecht, H., \& Gamer, M. (2010). Testing the egocentric mirror-rotation hypothesis. Seeing and Perceiving, 23(5-6), 373-383. http://dx.doi.org/10.1163/ $187847510 \times 540000$
Navon, D. (1987). Why do we blame the mirror for reversing left and right? Cognition, 27, 275-283. http://dx.doi.org/10.1016/S0010-0277(87)80012-4.

Pendergrast, M. (2003). Mirror/mirror. A history of the human love affair with reflection. New York: Basic Books.

Runeson, S. (1974). Constant velocity-not perceived as such. Psychological Research, 37 (1), 3-23.

Sareen, P., Ehinger, K. A., \& Wolfe, J. M. (2014). Through the looking-glass: Objects in the mirror are less real. Psychonomic Bulletin \& Reviewhttp://dx.doi.org/10.3758/s13423014-0761-8 (published on line 19 November 2014).

Savardi, U., \& Bianchi, I. (2014). Contraries in art: A glance at the structure of mirror reflections. Gestalt Theory, 36(3), 209-226.

Savardi, U., Bianchi, I., \& Bertamini, M. (2010). Naive prediction of orientation and motion in mirrors. From what we see to what we expect reflections to do. Acta Psychologica, 134(1), 1-15. http://dx.doi.org/10.1016/j.actpsy.2009.11.008.

Simons, D. J. (1996). In sight, out of mind: When object representations fail. Psychological Science, 7, 301-305. http://dx.doi.org/10.1111/j.1467-9280.1996.tb00378.x.

Tabata, T., \& Okuda, S. (2000). Mirror reversal simply explained without recourse to psychological processes. Psychonomic Bulletin \& Review, 7(1), 170-173. http://dx.doi.org/ 10.3758/BF03210737.

Takano, Y. (1998). Why does a mirror image look left-right reversed? A hypothesis of multiple processes. Psychonomic Bulletin \& Review, 5(1), 37-55. http://dx.doi.org/ 10.3758/BF03209456.

Troje, N. F., \& Kersten, D. (1999). Viewpoint-dependent recognition of familiar faces. Perception, 28, 483-487. http://dx.doi.org/10.1068/p2901.

Yates, J., Bessman, M., Dunne, M., Jertson, D., Sly, K., \& Wendelboe, B. (1988). Are conceptions of motion based on a naive theory or on prototypes? Cognition, 29(3), 251-275. 